\title{
Targeting BCL-2-expressing basal-like breast cancer with BH3-mimetics
}

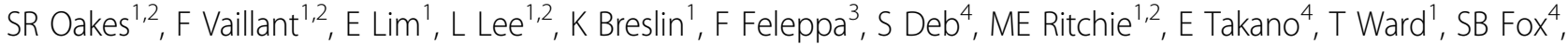 \\ D Generali ${ }^{5}$, GK Smyth ${ }^{1,2}$, A Strasser ${ }^{1,2}$, DCS Huang ${ }^{1,2}$, JE Visvader ${ }^{1,2}$, GJ Lindeman ${ }^{1,2,3^{*}}$ \\ From Familial Aspects of Cancer 2011 Research and Practice: A combined meeting of kConFab, Australian \\ Breast Cancer Family Study, Australian Colorectal Cancer Family Study, Australian Ovarian Cancer Study, \\ Family Cancer Clinics of Australia and New Zealand and kConFab \\ Kingscliff, Australia. 23-26 August 2011
}

Impairment of apoptosis is a hallmark of cancer and can result in resistance to chemotherapy. Tumour resistance to apoptosis is frequently acquired through deregulated expression of BCL-2 family members or inactivation of the p53 tumour suppressor pathway. Over-expression of the pro-survival protein BCL-2 is common in breast cancer (where it is readily detected by immunostaining), and has been shown to be an important prognostic marker. A potential role for BCL-2 as a therapeutic target in breast cancer, however, has not been explored. Recently, small molecules termed 'BH3-mimetics' have been developed that mimic the action of pro-apoptotic BH3only proteins. These bind and neutralize pro-survival proteins including BCL-2.

Tissue microarrays containing 197 primary breast tumours were evaluated for the expression of BCL-2, its anti-apoptotic relatives MCL-1 and BCL-XL, and the pro-apoptotic BH3-only ligand BIM. These proteins were co-expressed at relatively high levels in a substantial proportion of heterogeneous breast tumours, including clinically aggressive basal-like cancers. To determine whether the BH3-mimetic ABT-737 that neutralizes BCL-2, BCL-XL and BCL-W, had potential efficacy in targeting $\mathrm{BCL}$-2-expressing basal-like triple negative tumours, we generated a panel of primary breast tumour xenografts in immunocompromised mice and treated recipients with either ABT-737, docetaxel or a combination. Tumour response and overall survival were significantly improved by combination therapy, but only for tumour xenografts that expressed elevated levels of BCL-2. Treatment with ABT-737 alone was ineffective,

\footnotetext{
${ }^{1}$ The Walter and Eliza Hall Institute of Medical Research, Parkville VIC 3052, Australia

Full list of author information is available at the end of the article
}

suggesting that ABT-737 sensitizes the tumour cells to docetaxel. Combination therapy was accompanied by a marked increase in apoptosis and dissociation of BIM from BCL-2. Notably, BH3-mimetics also appeared effective in BCL-2-expressing xenograft lines that harbored p53 mutations.

In summary, primary breast tumour xenograft models that recapitulate the phenotype of the primary tumour have been developed as useful 'proof-of-principle', preclinical models. Our findings provide the first in vivo evidence that BH3-mimetics can be used to sensitize primary $\mathrm{BCL}-2$-expressing breast tumours to taxane chemotherapy. Our results suggest that elevated BCL-2 expression constitutes a predictive response marker in breast cancer. These findings provide a rationale for the development of clinical protocols using the oral analogue ABT-263 (navitoclax) as an adjunct to taxane chemotherapy in BCL-2-expressing basal-like and luminal breast cancer.

\section{Author details \\ ${ }^{1}$ The Walter and Eliza Hall Institute of Medical Research, Parkville VIC 3052, Australia. ${ }^{2}$ The University of Melbourne, Parkville, VIC 3010, Australia. ${ }^{3}$ The Royal Melbourne Hospital, Parkville VIC 3050, Australia. ${ }^{4}$ Peter MacCallum Cancer Centre, East Melbourne, VIC 3002, Australia. ${ }^{5}$ Ospitalieri di Cremona, Cremona 26100, Italy.}

Published: 12 April 2012

doi:10.1186/1897-4287-10-S2-A25

Cite this article as: Oakes et al:: Targeting BCL-2-expressing basal-like breast cancer with BH3-mimetics. Hereditary Cancer in Clinical Practice 2012 10(Suppl 2):A25.
C Biomed Central

C 2012 Oakes et al; licensee BioMed Central Ltd. This is an Open Access article distributed under the terms of the Creative Commons Attribution License (http://creativecommons.org/licenses/by/2.0), which permits unrestricted use, distribution, and reproduction in any medium, provided the original work is properly cited. 